\title{
Aplicación de un diseño experimental completamente al azar para determinar la variabilidad de tamaños en la síntesis de nanopartículas magnéticas de hierro.

\section{Application of a completely randomized experimental design to determine the} variability of sizes in the synthesis of magnetic nanoparticles of iron.

Francisco Salvador Vera Moreno ${ }^{1}$., Rosa Maricela Ormaza Hugo. ${ }^{2}$, Julio Rolando Coello Cabezas. ${ }^{3}$, Vilma Nohemí Yanchapanta Bastidas. ${ }^{4} \&$ Sara Esther Gusqui Macas ${ }^{5}$.

\begin{abstract}
.
DOI: https://doi.org/10.33262/cienciadigital.v2i4.1..195

This work applies a completely randomized experimental design within the process of synthesis of magnetic iron nanoparticles (magnetite- $\mathrm{Fe}^{2-}-\mathrm{Fe}^{3-}{ }_{2} \mathrm{O}_{4}$ ), with the objective of evaluating the size distribution. The synthesis of nanoparticles was carried out through the chemical co-precipitation method presented by Hidalgo et al., considering the modification of controllable parameters such as time, reagent concentration and temperature. The nanoparticles were characterized by infrared spectroscopy by Fourier transform (FT-IR) and scanning electron microscopy (SEM) with the support of the ImageJ software for the study of sizes. Through the analysis of variance, the hypothesis of equality of the mean $\mathrm{k}$ of the corresponding response variable is tested by means of the test statistic $\mathrm{F}$. The results show a value $\mathrm{p}=2,73 \mathrm{E}-12$ and with the pre-determined significance of 0,05 we reject the null hypothesis $\left(\mathrm{H}_{\mathrm{o}}\right)$, and it is accepted that at least a couple of treatments produce different sizes of NPMs. When comparing the difference in absolute value between the sample means with the value LSD $=0,00166487$, those differences that are greater than this value were declared significant.
\end{abstract}

Keywords: Magnetic nanoparticles, experimental design, analysis of variance, coprecipitation, LSD method.

\footnotetext{
Escuela Superior Politécnica de Chimborazo, Facultad de Ciencias, Riobamba, Ecuador. francissco@hotmail.com.

2 Escuela Superior Politécnica de Chimborazo. Facultad de Ciencias, Riobamba, Ecuador, rormaza@espoch.edu.ec

3 Escuela Superior Politécnica de Chimborazo. Facultad de Recursos Naturales, Riobamba, Ecuador, julio.coello@espoch.edu.ec

4 Escuela Superior Politécnica de Chimborazo. Facultad de Ciencias, Riobamba, Ecuador, vilma.yanchapanta@espoch.edu.ec

5 Escuela Superior Politécnica de Chimborazo. Facultad de Ciencias, Riobamba, Ecuador, sarahi05@live.com
} 


\section{Resumen.}

Este trabajo aplica un diseño experimental completamente al azar dentro del proceso de síntesis de nanopartículas magnéticas de hierro (magnetita- $\mathrm{Fe}^{2-} \mathrm{Fe}^{3-}{ }_{2} \mathrm{O}_{4}$ ), con el objetivo de evaluar la distribución de tamaños. La síntesis de nanopartículas se realizó a través del método de co-precipitación química presentado por Hidalgo et al., considerando la modificación de parámetros controlables como tiempo, concentración de reactivo y temperatura. Las nanopartículas fueron caracterizadas mediante espectroscopia infrarroja por transformada de Fourier (FT-IR) y microscopía electrónica de barrido (SEM) con el apoyo del software ImageJ para el estudio de tamaños. A través del análisis de varianza se prueba la hipótesis de igualdad de las k medias de la correspondiente variable respuesta mediante el estadístico de prueba F. Los resultados arrojan un valor $\mathrm{p}=2,73 \mathrm{E}-12$ y con la significancia prefijada de 0,05 rechazamos la hipótesis nula $\left(\mathrm{H}_{\mathrm{o}}\right)$, y se acepta que al menos un par de tratamientos producen distintos tamaños de NPMs. Al comparar la diferencia en valor absoluto entre las medias muestrales con el valor $\mathrm{LSD}=0,00166487$, se declaró significativas aquellas diferencias que son mayores a este valor.

Palabras clave: Nanopartículas magnéticas, diseño experimental, análisis de varianza, co-precipitación, método LSD.

\section{Introducción.}

La investigación del comportamiento de la materia en la escala nanométrica abre una prometedora perspectiva de nuevos conocimientos, las características físicas de las nanopartículas son muy distintas a las que se observan en un material de tamaño normal con la misma composición química. Hoy en día, se comienzan a utilizar de modo habitual, técnicas experimentales que permiten fabricar, caracterizar y manipular éstas partículas de tamaño minúsculo (Grande, 2007). En general, las propiedades de las nanopartículas magnéticas (NPMs), dependen en gran medida del método de síntesis que se utilice y de la estructura química resultante.

Las NPMs presentan diversas propiedades, entre las cuales podemos mencionar su tamaño, el cual las permite ser comparables a virus o ciertas proteínas; su facilidad al momento de ser manipuladas con gradientes de campo magnético externo, y además la superficie que tienen y la cual puede ser modificada adecuadamente para trabajar en el análisis de agentes biológicos (Tartaj et al., 2005).

En la mayoría de los casos de síntesis se espera un intervalo de tamaño de las partículas que va desde 1 a $100 \mathrm{~nm}$ pudiendo mostrar propiedades características de los óxidos de Hierro, como es el superparamagnetismo (Briones, 2016). 
Los métodos de síntesis químicos, como la co-precipitación, presentan varias ventajas sobre otros métodos por su bajo costo y su rápida obtención de grandes poblaciones de NPMs (Fernández., 2013). El análisis de éste método permite observar que ciertos parámetros controlables pueden ser manipulados, y de esta manera tratar de optimizar una receta estándar; con el objetivo de determinar cómo va a afectar la modificación de estos parámetros al tamaño de las NPMs, ya que, de esto dependerán sus propiedades (Castrillón, 2012).

El diseño experimental completamente al azar, tiene la ventaja de ser el más simple de todos los diseños que se utilizan para comparar dos o más tratamientos, dado que para éste sólo se consideran dos fuentes de variabilidad: los tratamientos y el error aleatorio (Gutierrez \& De La Vara, 2010).

En esta investigación, se buscó estudiar los parámetros más representativos de la muestra, y determinar la existencia de variaciones en el tamaño de las NPMs. Mediante el método de coprecipitación se sintetizaron las nanopartículas, las cuales se caracterizaron mediante algunas técnicas instrumentales, con lo cual se obtuvo valores de los tamaños de las NPMs (López et al., 2014). Se utilizó el modelo LSD, para comparar los tratamientos y verificar si estos son pertinentes o no de ser realizados, es decir, buscamos identificar los tratamientos más representativos hacia el tamaño de nuestras muestras (MPNS).

En esta investigación, se estudió el proceso de síntesis de NPMs, las cuales fueron obtenidas por medio del método químico de co-precipitación; además con la aplicación del modelo estadístico totalmente al azar se buscó determinar si los tratamientos planteados afectan en el tamaño de las NPMs.

\section{Materiales y métodos.}

\section{Síntesis de nanopartículas.}

La síntesis de NPMs se realizó por medio del proceso de co-precipitación química, bajo la modalidad de adsorción superficial, por las ventajas que este presenta en el proceso de obtención (Cuadrado, 2017) (Lu et al., 2007).

Se tomó como base la receta estándar presentada por Hidalgo et al. En una bureta de $10 \mathrm{ml}$ se fijaron 7,1 ml de agua destilada, para luego trasvasar a un vaso de precipitación de $10 \mathrm{ml}$. En un vaso de precipitación se agrega 177,5 mg de $\mathrm{FeCl}_{3} \cdot 6 \mathrm{H}_{2} \mathrm{O}$ y 88,75 gr $\mathrm{Na}_{2} \mathrm{SO}_{3}$; se agita con una varilla hasta tener una mezcla homogénea y de color rojizo. Posterior a esto se agrega $0,35 \mathrm{ml}$ de ácido oleico y se coloca en un agitador-calentador magnético, durante 7 minutos, hasta obtener una temperatura de $55^{\circ} \mathrm{C}$ y $5000 \mathrm{rpm}$. Luego se agrega $60 \mathrm{ml} \mathrm{de} \mathrm{Na}(\mathrm{OH})$ y se mantiene la agitación magnética y la temperatura por una hora, logrando que se combinen todos los elementos de las NPMs.

Para la purificación de la muestra se empleó un imán de neodimio de campo magnético de 0,2 $\mathrm{T}$, atrayendo las partículas hacia el fondo del vaso de precipitación para la posterior remoción del sobrenadante. Se realizan varios lavados con agua destilada de las NPMs.

Las muestras obtenidas de material nanoparticulado, fueron caracterizadas por medio de: espectroscopia infrarroja por transformada de Fourier (FT-IR), permitiendo verificar la 
presencia de magnetita en cada una de las muestras, y microscopía electrónica de barrido (SEM) para identificar las propiedades morfológicas de las poblaciones de NPMs de hierro.

\section{Análisis Estadístico.}

Para determinar la variabilidad en el tamaño de las NPMs, se establecieron como parámetros controlables: la concentración del reactivo, la temperatura de ebullición y el tiempo de agitación. En función de estos parámetros, se constituyeron 10 tratamientos considerando límites superiores e inferiores (tabla 1). Para la concentración se estableció un límite superior e inferior de 98 y $80 \mathrm{mg}$ respectivamente; mientras que, para la temperatura se tomó como límite inferior $35^{\circ} \mathrm{C}$ y como límite superior $80{ }^{\circ} \mathrm{C}$. El tiempo de agitación se relacionó directamente con la temperatura, tomando tiempos lo suficientemente cortos para que la muestra no se evapore en su totalidad y así se puedan combinar todos los reactivos.

Tabla 1. Tratamientos y Parámetros controlados en las muestras de NPMs.

\begin{tabular}{cccc}
\hline Parámetro & $\begin{array}{c}\text { Concentración de } \\
\text { Reactivo }(\mathrm{mg})\end{array}$ & $\begin{array}{c}\text { Tiempo de } \\
\text { Agitación }(\mathrm{min})\end{array}$ & $\begin{array}{c}\text { Temperatura de } \\
\text { Ebullición }\left({ }^{\circ} \mathrm{C}\right)\end{array}$ \\
\hline $\mathrm{T} 1$ & 80 & 3 & 35 \\
\hline $\mathrm{T} 2$ & 82 & 4 & 40 \\
\hline $\mathrm{T} 3$ & 84 & 5 & 45 \\
\hline $\mathrm{T} 4$ & 86 & 6 & 50 \\
\hline $\mathrm{T} 5$ & 88 & 7 & 55 \\
\hline $\mathrm{T} 6$ & 90 & 8 & 60 \\
\hline $\mathrm{T} 7$ & 92 & 9 & 65 \\
\hline $\mathrm{T} 8$ & 94 & 10 & 70 \\
\hline $\mathrm{T} 9$ & 96 & 11 & 85 \\
\hline $\mathrm{T} 10$ & 98 & 12 &
\end{tabular}

Mediante el diseño completamente al azar se compara las poblaciones de NPMs mediante la hipótesis de igualdad de medias. Para lo cual, se considera como factor de estudio los tratamientos que están formados en función de los parámetros controlables (la concentración del reactivo, la temperatura de ebullición y el tiempo de agitación), obteniendo como variable respuesta el tamaño de las NPMs (Medero, 2002). Si los tratamientos propuestos tienen un efecto el tamaño de las NPMs se puede describir con el modelo estadístico lineal (1), lo que implica que el diseño completamente al azar actúa en dos fuentes de variabilidad: los tratamientos y el error aleatorio. Posteriormente se realiza un análisis de comparación entre tratamientos utilizando el método LSD, para determinar cuáles de los 10 tratamientos utilizados en el experimento son significativamente diferentes.

Para conseguir un valor representativo, cada muestra tuvo 100 datos que comprueben los tamaños de las NPMs.

\section{Diseño experimental.}

Ho: $\mu 1=\mu 2=\mu 3=\mu 4=\mu 5=\mu 6=\mu 7=\mu 8=\mu 9=\mu 10$

$\mathrm{H} 1$ : No todas las medias son iguales al menos 2 son diferentes.

\section{Modelo Estadístico Lineal.}




$$
Y_{i j}=\mu-\alpha_{i}-\varepsilon_{i j}
$$

Dónde:

$Y_{i j}$ : es el j-ésimo Tamaño de las NPMs en el i-ésimo tratamiento.

$\mu$ : es el Tamaño de las NPMs.

$\alpha_{i}$ : es el efecto del i-ésimo Tratamiento en el Tamaño de las NPMs.

$\varepsilon_{i j}$ : es el error aleatorio asociado a la medición del j-ésimo tamaño de las NPMs en el i-ésimo tratamiento.

Para que los resultados obtenidos en el análisis de varianza tengan validez deben cumplir con los supuestos del modelo:

* $\mathrm{k}$ poblaciones normales con media $\mu 1, \ldots, \mu \mathrm{k}$.

* Las k poblaciones tienen varianza constante.

* Las k poblaciones son independientes.

Mediante el uso del programa R, se realizó el test de Shapiro-Wilk para normalidad. Para el supuesto de Homogeneidad se utilizó la Prueba de Bartlett, considerando las hipótesis:

$$
H_{0}: \sigma_{1}^{2}=\sigma_{2}^{2}=\cdots=\sigma_{k}^{2} \quad H_{1}: \sigma_{i}^{2} \neq \sigma_{j}^{2} \quad i \neq j
$$

Para el supuesto de independencia se usó como método analítico el contraste de DurbinWatson. Y una vez que, se verificó el cumplimiento de los supuestos del modelo estadístico, se realizó un ANOVA para establecer la existencia o no, del efecto de los tratamientos en el tamaño de las NPMs. También se utilizó el Método LSD para comparar los tratamientos y determinar cuáles son estadísticamente diferentes (Gutierrez \& De La Vara, 2010).

\section{Resultados.}

\section{Caracterización de Nanopartículas Magnéticas de Hierro.}

Mediante el uso de Espectroscopia FT- IR, se determinó la afectación de los parámetros controlables en la síntesis, esto se puede observar en la figura 1, donde el tratamiento 10 muestra una notable intensidad espectral, mientras que, el tratamiento 5 expone una intensidad similar a la reportada por Gómez, en el 2011 (Gómez et al, 2011). Además, pese a que el tratamiento 1 exhibe una intensidad baja, ésta presenta picos característicos de la magnetita (Urquijo, 2007). 


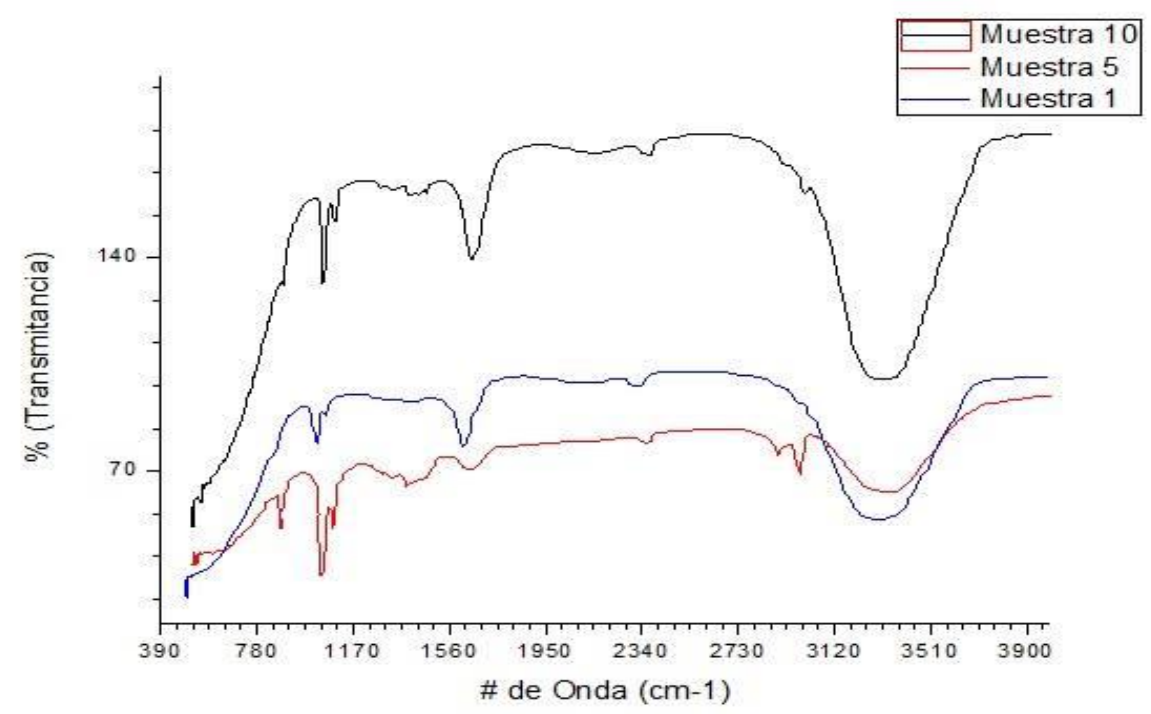

Figura 1. Intercomparación de espectros de los tratamientos. Tratamiento 1 (80mg, $3 \mathrm{~min}$, $\left.35^{\circ} \mathrm{C}\right)$; Tratamiento $5\left(88 \mathrm{mg}, 7 \mathrm{~min}, 55^{\circ} \mathrm{C}\right)$; Tratamiento $10\left(98 \mathrm{mg}, 12 \mathrm{~min}, 80^{\circ} \mathrm{C}\right)$.

Mientras que con el uso del Microscopio Electrónico de Barrido (SEM por sus siglas en inglés), se obtuvo imágenes claras de las poblaciones de NPMs. En estas imágenes se considerada 100 NPMs de cada tratamiento, para establecer la variación de tamaño según la manipulación de parámetros controlables, la Figura 2, según los tratamientos T1 (bajo), T5 (medio) y T10 (alto), revela que cuando mayor fue la concentración de reactivo, temperatura y tiempo de agitación empleados, mayor fue la cantidad de NPMs iguales o menores a 29 nm.

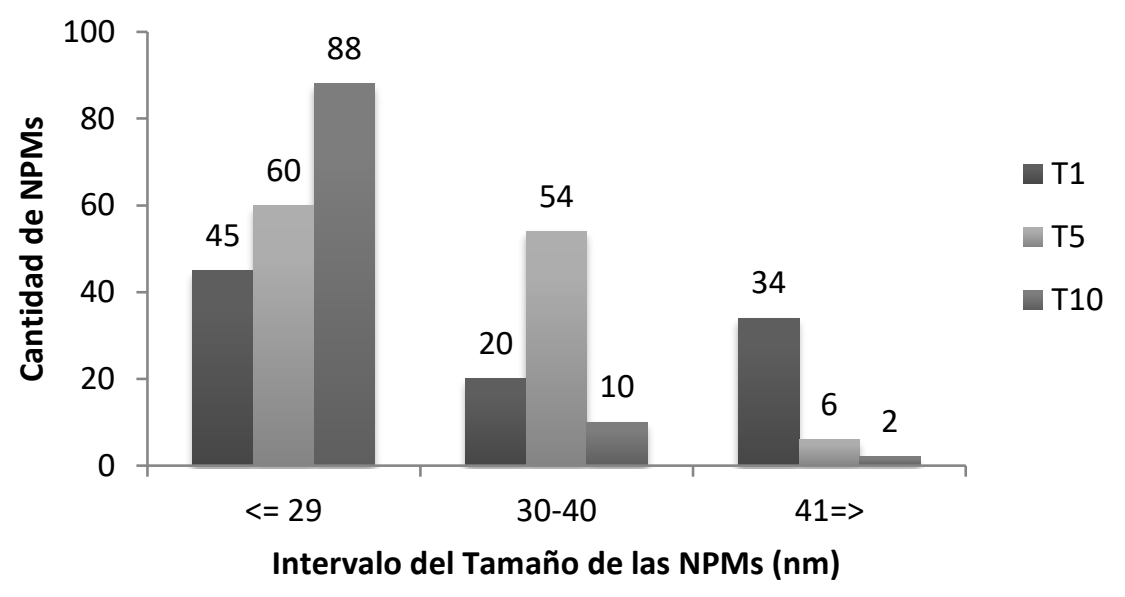

Figura 2. Población de NPMs en intervalos de tamaños.

\section{Análisis Estadístico.}

Mediante el método analítico de Shapiro-Wilk se analizó la normalidad de los residuos, obteniendo un valor estadístico $\mathrm{W}=0,9757$ con un $\mathrm{p}$-value $=0,704$. Como $\mathrm{p}$-value $>0,05$ se concluye que los datos proceden de una distribución normal y presenta un nivel de confianza del 95\%. La Figura 3, muestra que se cumple la normalidad ya que los residuos provienen de una distribución normal. 


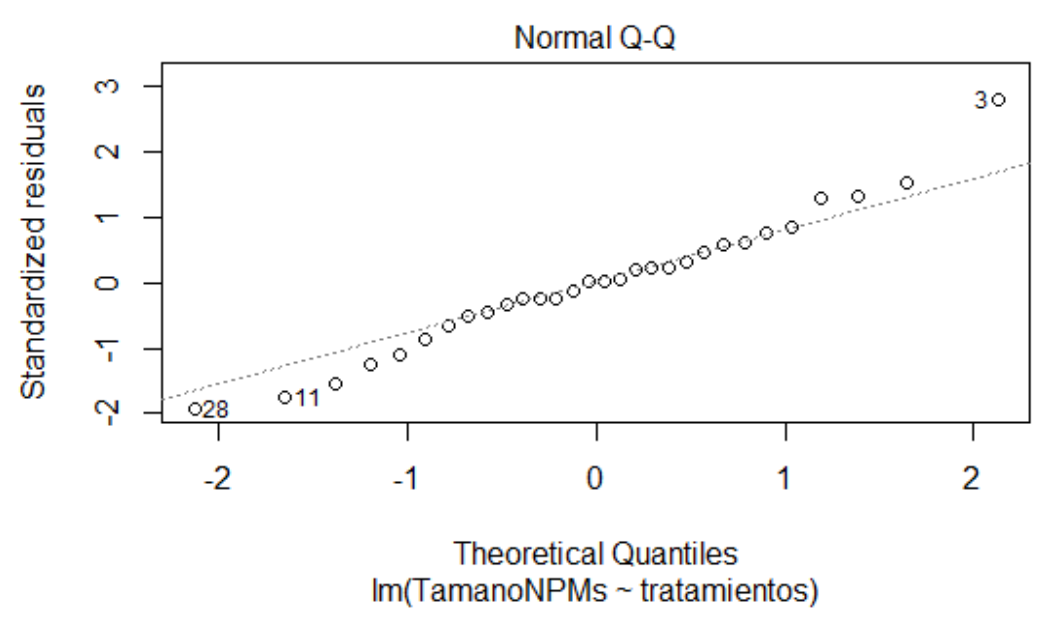

Figura 3. Distribución normal de tratamientos.

En la Figura 4, se presenta los resultados de la prueba de Bartlett para homogeneidad de varianzas, se obtuvo el valor Bartlett's K-squared de 17,402 y un p-value=0,06278, lo que nos indica homogeneidad en los datos.

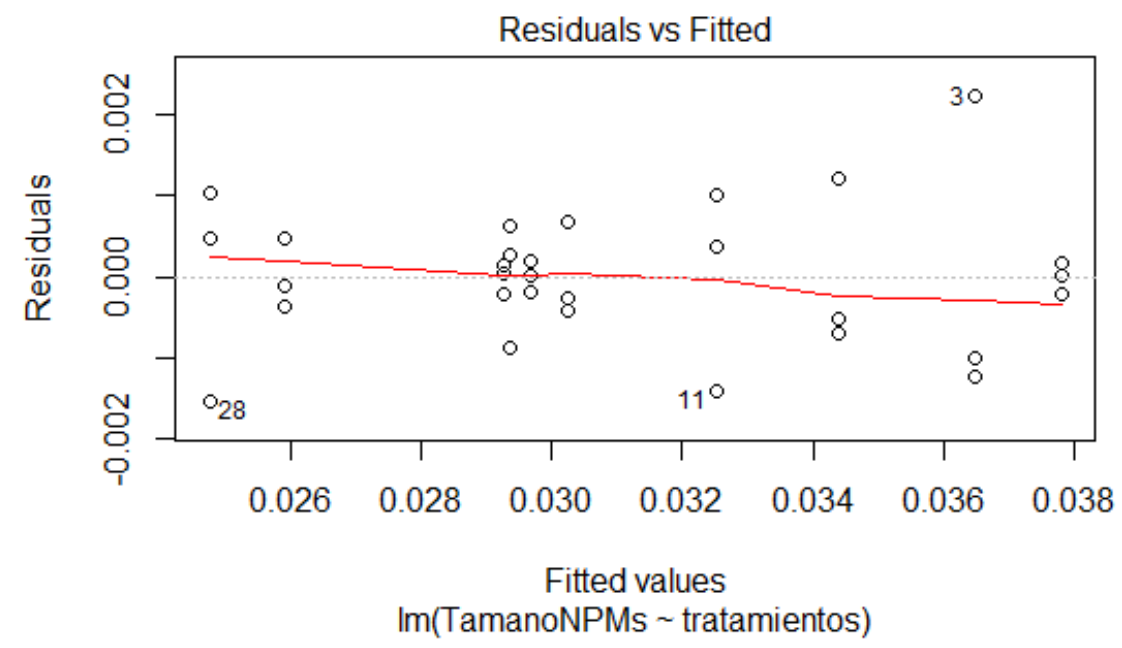

Figura 4. Homogeneidad de Varianzas de tratamientos.

La prueba de Durbin-Watson arroja un valor $\mathrm{DW}=2,4819$ y un $\mathrm{p}$-value $=0,3336$, lo que muestra que los datos son independientes, es decir no existe autocorrelación, esto se presenta en la Figura 5.

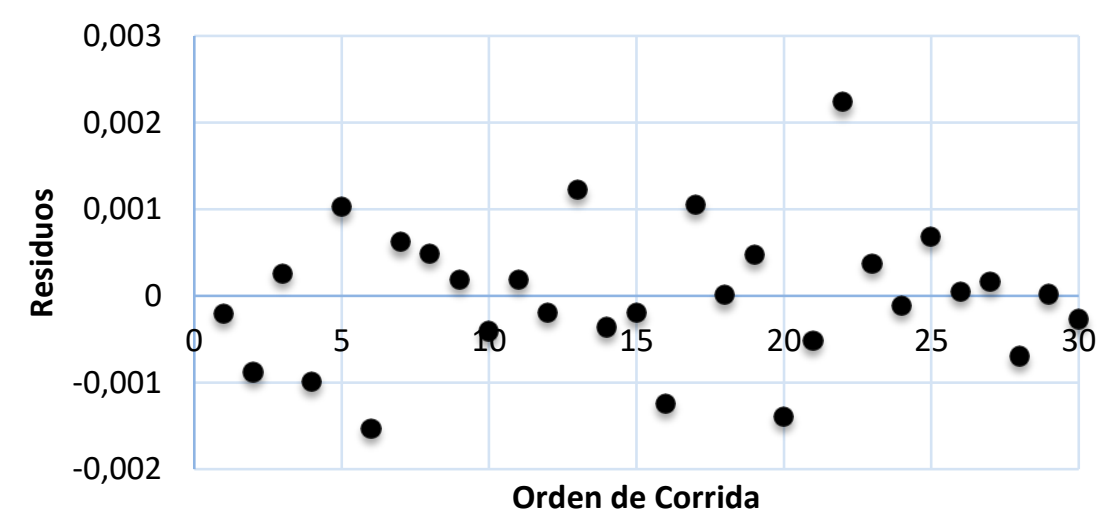

Figura 5. Independencia de la variable aleatoria residuos. 
Luego de establecer el cumplimiento de todos los supuestos, comprobando entonces, que los parámetros del modelo tienen criterios de calidad, se realizó un ANOVA (tabla 2). En el análisis de varianza para determinar si los tratamientos muestran diferencias significativas, se obtuvo un valor $\operatorname{Pr}(>\mathrm{F})$ de 2,74E-12, que siendo menor que el nivel de significancia de 0,05, decimos que el factor Tratamiento tiene un efecto en el tamaño de las NPMs de Fe (variable respuesta).

Tabla 2.Análisis de Varianza

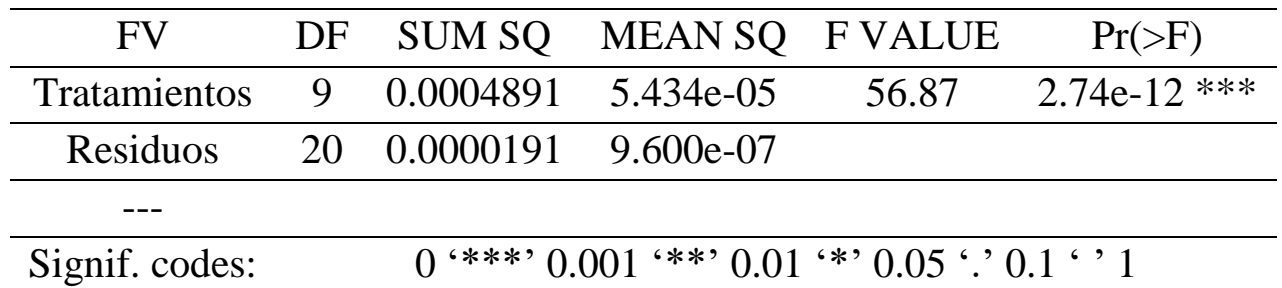

La prueba de rangos múltiples o comparaciones, realizada a través del Método LSD, arrojó un valor de 0,00166487, que comparado con las medias de cada uno de los tratamientos, determina cuáles son estadísticamente diferentes. La tabla 3, muestra que de las 45 combinaciones realizadas entre tratamientos, únicamente entre T1-T2, T10-T9, T5-T6, T5T7, T5-T8, T6-T7, T6-T8 y T7-T8, no existe diferencia estadística significativa.

Tabla 3.Análisis de Comparación por el Método LSD

\begin{tabular}{cccccc}
\hline \multicolumn{2}{l}{ Tratamientos } & Diferencia & pvalue & signif \\
\hline $\mathrm{T} 1$ & - & $\mathrm{T} 10$ & $1,17 \mathrm{E}-04$ & 0.0000 & $* * *$ \\
\hline $\mathrm{T} 1$ & - & $\mathrm{T} 2$ & $-1,33 \mathrm{E}-03$ & 0.1121 & \\
\hline $\mathrm{T} 1$ & - & $\mathrm{T} 3$ & $2,08 \mathrm{E}-03$ & 0.0168 & $*$ \\
\hline $\mathrm{T} 1$ & - & $\mathrm{T} 4$ & $3,96 \mathrm{E}-03$ & 0.0001 & $* * *$ \\
\hline $\mathrm{T} 1$ & - & $\mathrm{T} 5$ & $7,12 \mathrm{E}-03$ & 0.0000 & $* * *$ \\
\hline $\mathrm{T} 1$ & - & $\mathrm{T} 6$ & $6,23 \mathrm{E}-03$ & 0.0000 & $* * *$ \\
\hline $\mathrm{T} 1$ & - & $\mathrm{T} 7$ & $6,81 \mathrm{E}-03$ & 0.0000 & $* * *$ \\
\hline $\mathrm{T} 1$ & - & $\mathrm{T} 8$ & $7,22 \mathrm{E}-03$ & 0.0000 & $* * *$ \\
\hline $\mathrm{T} 1$ & - & $\mathrm{T} 9$ & $1,06 \mathrm{E}-04$ & 0.0000 & $* * *$ \\
\hline $\mathrm{T} 10$ & - & $\mathrm{T} 2$ & $-1,30 \mathrm{E}-04$ & 0.0000 & $* * *$ \\
\hline $\mathrm{T} 10$ & - & $\mathrm{T} 3$ & $-9,63 \mathrm{E}-03$ & 0.0000 & $* * *$ \\
\hline $\mathrm{T} 10$ & - & $\mathrm{T} 4$ & $-7,75 \mathrm{E}-03$ & 0.0000 & $* * *$ \\
\hline $\mathrm{T} 10$ & - & $\mathrm{T} 5$ & $-4,59 \mathrm{E}-03$ & 0.0000 & $* * *$ \\
\hline $\mathrm{T} 10$ & - & $\mathrm{T} 6$ & $-5,48 \mathrm{E}-03$ & 0.0000 & $* * *$ \\
\hline $\mathrm{T} 10$ & - & $\mathrm{T} 7$ & $-4,90 \mathrm{E}-03$ & 0.0000 & $* * *$ \\
\hline $\mathrm{T} 10$ & - & $\mathrm{T} 8$ & $-4,49 \mathrm{E}-03$ & 0.0000 & $* * *$ \\
\hline $\mathrm{T} 10$ & - & $\mathrm{T} 9$ & $-1,14 \mathrm{E}-03$ & 0.1698 & \\
\hline $\mathrm{T} 2$ & - & $\mathrm{T} 3$ & $3,41 \mathrm{E}-03$ & 0.0004 & $* * *$ \\
\hline $\mathrm{T} 2$ & - & $\mathrm{T} 4$ & $5,28 \mathrm{E}-03$ & 0.0000 & $* * *$ \\
\hline $\mathrm{T} 2$ & - & $\mathrm{T} 5$ & $8,45 \mathrm{E}-03$ & 0.0000 & $* * *$
\end{tabular}


Vol. 2, N4.1, p. 140-153, octubre - diciembre, 2018

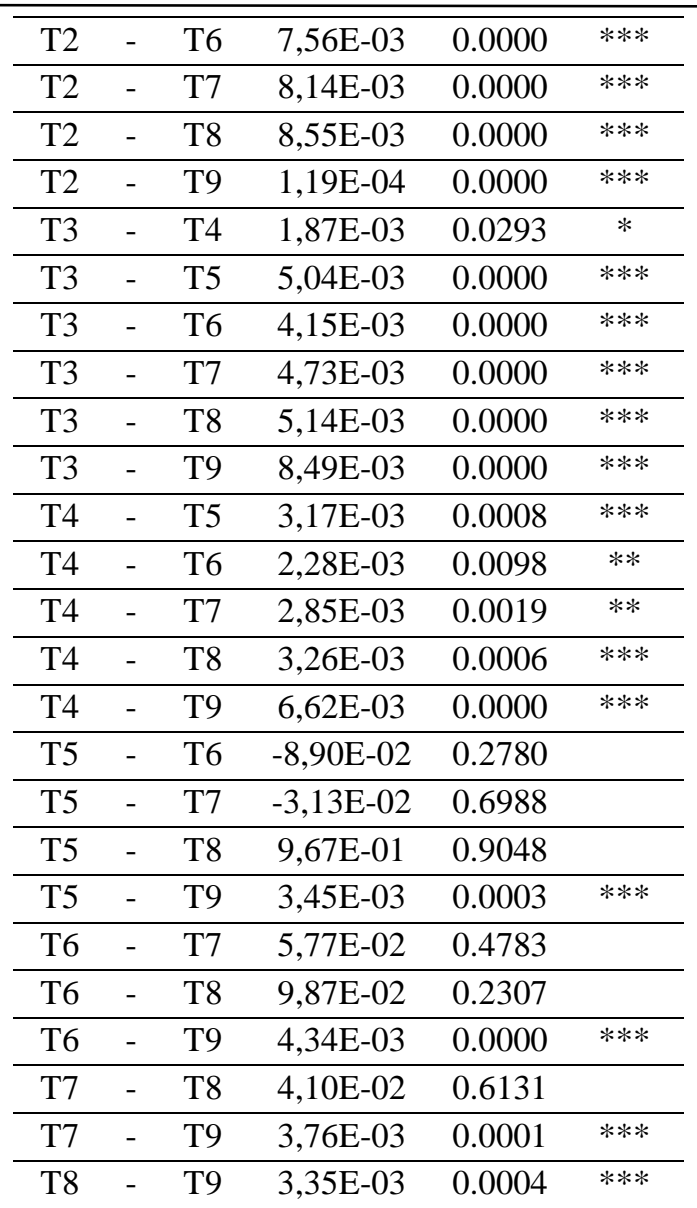

\section{Discusión.}

En el esquema en cascada presentado en la Figura 1, de los tratamientos 1, 5 y 10, se puede evidenciar una notable intensidad espectral en la muestra del T10; debido a que ésta tiene mejores características posteriores a su síntesis y un bajo nivel de oxidación (Kadakia., 2008). Mientras que la muestra del T5, que corresponde al estándar, expone una intensidad similar a las presentadas en otras investigaciones (Gómez, 2011). La similitud en los resultados en éste tratamiento, nos permite demostrar que con la aplicación adecuada del método, en este caso, la manipulación de las condiciones de síntesis, se puede permitir el control racional de la morfología de las partículas (Zanella, 2012). Finalmente, la muestra correspondiente al T1, presenta nivel de intensidad más bajo, pero a pesar de esto se pudieron visualizar los picos de absorción característicos de la magnetita, pero con intensidades menores en sus componentes (Morales, 2007).

El SEM nos proporcionó imágenes claras de la cristalización de las NPMs magnéticas, donde se pudo observar la aglomeración de las NPMs, evidenciando su naturaleza magnética, típica de este tipo de nanocompuestos (Fernández, 2013; Daniel \& Astruc, 2004). Además, se exhibió la pérdida de su propiedad de fluidez al momento de ser analizadas en el SEM, debido a la evaporación del medio portador. Las muestras analizadas a una distancia de $17 \mathrm{~mm}$, con un aumento de $100 \mu \mathrm{m}$ y una energía de $10 \mathrm{KV}$, presentaron la formación de cristales de magnetita con tamaños variables como los expuestos en la figura 2. Sin embargo, al tener altas 
poblaciones de NPMs menores a $29 \mathrm{~nm}$ y muy pocas superiores a $41 \mathrm{~nm}$, bajo las condiciones de diseño experimental aplicadas, se puede deducir la elevada incidencia del método; además de las propiedades superparamagnéticas que estas adquieren cuando su tamaño es menor a 30 nm (Luciano et al., 2013).

La variación de tamaños indicada, se puede explicar por las condiciones del tiempo de agitación, lo que provoca que no exista uniformidad en el proceso de síntesis, además, los niveles de temperatura propician la acción del surfactante sobre las NPMs y que cause posibles uniones entre sí.

El cumplimiento de los supuestos, presentados en los resultados, donde, los errores son independientes (Figura 3); los errores están normalmente distribuidos con media cero y varianza constante (Figura 4.); y de homogeneidad de varianza entre los tratamientos (Figura 5), validaron el análisis de varianza realizado. Esto indica que, el material experimental fue homogéneo y que la aplicación de la aleatorización fue apropiada (Castillo et al., 2011).

El análisis de varianza (ANOVA) mostrado en la tabla 3, indica que predomina la variabilidad debida a los tratamientos respecto a la variabilidad debida al error (Gutiérrez \& De la Vara, 2008). Entonces, con el diseño del experimento completamente al azar, para determinar si la variación de tamaños (variable respuesta), depende del tratamiento, se identifica que estos tienen efecto, es decir, las medias son diferentes. Entonces, la necesaria aplicación de la prueba de rangos múltiples, captó la diferencia mínima entre los tratamientos; pudiendo determinar que existe un $74 \%$ de alta diferencia estadística significativa de las 45 comparaciones realizadas.

\section{Conclusiones .}

- Las Nanopartículas magnéticas (NPMs), fueron sintetizadas con éxito mediante el método de co-precipitación química bajo la modalidad de adsorción superficial, y se las caracterizó con el uso de técnicas espectroscópicas FT-IR, que permitió determinar la presencia de magnetita en función de los espectros obtenidos; y con el Microscopio electrónico de barrido (SEM), se pudo ver la estructura esférica tipo núcleo-corona, las poblaciones de tamaños y la composición química de las mismas.

- Para el análisis estadístico se incorporaron distintos procesos químicos, y se incluyó la variación de parámetros controlables como la temperatura, tiempo de agitación y concentración de reactivos; tomando en cuenta a los tratamientos (alto medio y bajo). Con esto se pudo visualizar la relación directa que existe entre las propiedades físicas y químicas de las NPs.

- Se determinó que existe un alto grado de diferencia estadística significativa entre cada uno de los tratamientos estudiados, lo cual es de gran importancia al momento de analizar el tamaño de las nanopartículas magnéticas de hierro. 


\section{Referencias bibliográficas.}

Briones, P. R. (12 de Junio de 2016). Nanopartículas Magneticas para tratamiento y Diagnóstico de Cáncer. Madrid - España. Obtenido de https://www.google.com/url? sa=t\&rct=j\&q=\&esrc=s\&source=web\&cd=1\&cad=rja \&uact $=8 \&$ ved $=0$ ahUKEwj4lfingOjaAhXqxlkKHcyUBKgQFgguMAA\&url=http $\% 3$ A\%2F\%2F147.96.70.122\%2FWeb\%2FTFG\%2FTFG\%2FMemoria\%2FPAULA\%25 20RUIZ\%2520BRIONES.pdf\&usg=AOvVaw0sr9LfzNTazXGXs6yxjQ-R

Castillo, C., Mejía C., \& Arévalo J. (2011). Diseño de Experimentos al Completo Azar. Estadística, Matemática y Computación. Obtenido de http://reyesestadistica.blogspot.com/2011/07/diseno-de-experimentosalcompletoazar.html

Castrillón, M. (2012). Síntesis de nanopartículas magnéticas y su aplicación en nanocompuestos de matriz polimérica con propiedades magnéticas. Departamento de Ingeniería Química y Tecnologías del Medio Ambiente. Universidad de Zaragoza.

Cuadrado, C. (2017). Repositorios ESPOCH. Recuperado el 12 de Junio de 2018, de http://dspace.espoch.edu.ec/handle/123456789/6912

Daniel, M.-C., \& Astruc, D. (2004). Gold nanopartilces: Assembly, supramolecular chemistry, quantum-size-related properties, and applications toward biology, catalysis and nanotechnology. Vol. 104 (1): 293-346. Chemical Reviews.

Fernández., K. C. (2013). Síntesis y caracterización de nanopartículas magnéticas. Centro de Investigaciones en óptica. Argentina.

Gómez López, P., González V, Garza M., \& Esquive R. (2017). Síntesis y caracterización de nanocompósitos de óxido de hierro en un polimero semiconductor. Vol. 20(77):57-65. Ingenierías.

Grande, A. H. (2007). Nanotecnología y nanopartículas magnéticas: la física actual en lucha contra la enfermedad. Vol. 101(2): 321-327. Rev.R.Acad.Cienc. Exact.Fís.Nat. España.

Gutiérrez P., H. G. \& De la Vara, R. (2008). Análisis y diseño de experimentos. 2a Ed. Mexico: Mc Graw Hill.

Kadakia, K. (2008). Removal of Arsenic Contamination from Water Using Magnetite Nanoparticles. The National High School Journal of Science. Retrieved from http://nhsjs.com/2012/removal-of-arsenic-contamination-from-water-usingmagnetite-nanoparticles/ 
López, J. L., Dias J. H., Paniago R., Paniago R., D. Pfannes H., \& Balzuweit K. (2014). Progresos en la preparación de nanopartículas magnéticas multifuncionales para aplicación en la medicina. Vol. 10(2): 5-13. Revista ECI, Perú.

Lu, A. H., Salabas, E. L., Schuth, F. (2007). Magnetic nanoparticles: Synthesis, protection, functionalization, and applications. Angewandte Chemie Int. Ed., 46, 1222-1244.

Luciano-Carlos, F., García-Einschlag, M., \& González-Mártire, D. (2013), Applications of Magnetite Nanoparticles for Heavy Metal Removal from Wastewater. Waste WaterTreatment Technologies and Recent Analytical Developments. Retrieved from http://cdn.intechopen.com/pdfs/41219/InTech-

Applications_of_magnetite_nanoparticles_for_heavy_metal_removal_from_wastewa ter.pdfMedero, M. A. (2002). Curso-Taller Estadística Aplicada a la Investigación. Nayarit.

Urquijo M., J. P. (2007). Síntesis de Nanopartículas Magnéticas y su Implementación en los Ferrofluidos. Universidad de Antioquia. Facultad De Ciencias Exactas y Naturales. Medellín.

Tartaj, P., Morales, M., González-Carreño, T., Veintemillas-Verdaguer, S., \& Serna, C. (2005). Advances in magnetic nanoparticles for biotechnology applications. Journal of Magnetism and Magnetic Materials, 290, 28-34.

Zanella R. (2012). Metodologías para la síntesis de nanopartículas: controlando forma y tamaño. México. Mundo Nano. Vol.5 (1): 69-81. 


\section{Para citar el artículo indexado.}

Vera F., Ormaza R., Coello J., Yanchapanta V. \& Gusqui S. (2018). Aplicación de un diseño experimental completamente al azar para determinar la variabilidad de tamaños en la síntesis de nanopartículas magnéticas de hierro. Revista electrónica Ciencia Digital 2(4), 140-153. Recuperado desde: http://cienciadigital.org/revistacienciadigital2/index.php/CienciaDigital/article/view/195/173

\section{Ciencia \\ LDigital}

El artículo que se publica es de exclusiva responsabilidad de los autores y no necesariamente reflejan el pensamiento de la Revista Ciencia Digital.

$\mathrm{El}$ articulo queda en propiedad de la revista y, por tanto, su publicación parcial y/o total en otro medio tiene que ser autorizado por el director de la Revista Ciencia Digital.
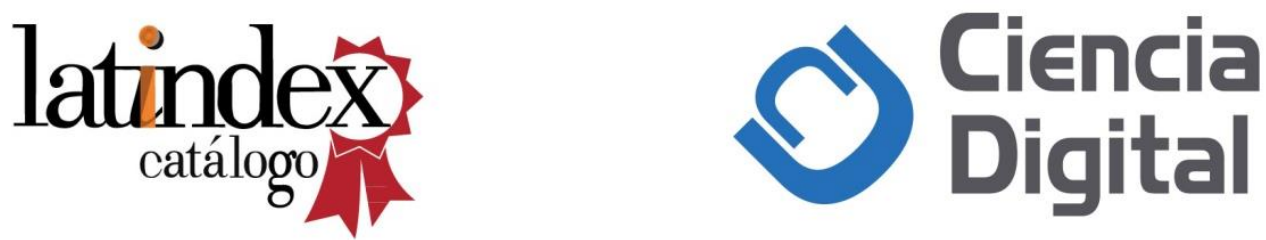